\title{
EXPLICIT SERIES SOLUTION OF A CLOSURE MODEL FOR THE VON KÁRMÁN-HOWARTH EQUATION
}

\author{
ZENG LIU ${ }^{1}$, MARTIN OBERLACK ${ }^{2,3,4}$, VLADIMIR N. GREBENEV ${ }^{5}$ and \\ SHI-JUN LIAO ${ }^{\otimes 1}$
}

(Received 30 May, 2010; revised 2 April, 2011)

\begin{abstract}
The homotopy analysis method (HAM) is applied to a nonlinear ordinary differential equation (ODE) emerging from a closure model of the von Kármán-Howarth equation which models the decay of isotropic turbulence. In the infinite Reynolds number limit, the von Kármán-Howarth equation admits a symmetry reduction leading to the aforementioned one-parameter ODE. Though the latter equation is not fully integrable, it can be integrated once for two particular parameter values and, for one of these values, the relevant boundary conditions can also be satisfied. The key result of this paper is that for the generic case, HAM is employed such that solutions for arbitrary parameter values are derived. We obtain explicit analytical solutions by recursive formulas with constant coefficients, using some transformations of variables in order to express the solutions in polynomial form. We also prove that the Loitsyansky invariant is a conservation law for the asymptotic form of the original equation.
\end{abstract}

2000 Mathematics subject classification: primary 34B15; secondary 22E70.

Keywords and phrases: homotopy analysis method, von Kármán-Howarth equation, solutions in closed form, conservation law.

\section{Introduction}

In this paper, we deal with a nonlinear problem arising in homogeneous isotropic turbulence. The physical problem imposes a particular form of a closure model [21] on

\footnotetext{
${ }^{1}$ State Key Lab of Ocean Engineering, School of Naval Architecture, Ocean and Civil Engineering, Shanghai Jiao Tong University, 800 Dongchuan Road, Shanghai 200240, PR China; e-mail: z_liu@sjtu.edu.cn, sjliao@sjtu.edu.cn.

${ }^{2}$ Chair of Fluid Dynamics, Department of Mechanical Engineering, Technische Universität Darmstadt, Petersenstr. 30, 64287 Darmstadt, Germany.

${ }^{3}$ Center of Smart Interfaces, TU Darmstadt, Petersenstr. 32, 64287 Darmstadt, Germany.

${ }^{4}$ GS Computational Engineering, TU Darmstadt, Dolivostr. 15, 64293 Darmstadt, Germany; e-mail: oberlack@fdy.tu-darmstadt.de.

${ }^{5}$ Institute of Computational Technologies, Russian Academy of Science, Lavrentiev Ave. 6, Novosibirsk 630090, Russia; e-mail: vngrebenev@gmail.com.

(C) Australian Mathematical Society 2011, Serial-fee code 1446-1811/2011\$16.00
} 
the von Kármán-Howarth equation which then leads to the task of solving a nonlinear parabolic partial differential equation (PDE).

Emphasis is placed on the use of the homotopy analysis method (HAM) [11] to find solutions of the nonlinear problem for the normalized longitudinal two-point double velocity correlation [19]. The HAM was suggested by Liao to obtain series solutions of nonlinear differential equations. Unlike other methods such as explicitly constructing integrating factors [25], the HAM is based on homotopy, a fundamental concept in topology and differential geometry. Briefly, one constructs a continuous mapping of an initial guess approximation to the exact solution of the equations. An auxiliary linear operator is chosen to construct the continuous mapping, and an auxiliary parameter is used to ensure the convergence of the solution series.

Unlike perturbation techniques, this approach is independent of any small or large physical parameters, and is thus valid whether a nonlinear problem contains small or large physical parameters or not. Besides this, the HAM provides great freedom to choose proper base functions to approximate a nonlinear problem. Most importantly, it provides a simple way to adjust and control the convergence of the solution series.

The paper is organised as follows. The aforementioned nonlinear problem for the parabolic PDE is described in Section 2. This equation is the lowest-order two-point statistical equation for turbulence dynamics, derived from the Navier-Stokes equations under the assumption of homogeneity and isotropy of a turbulent flow. In the limit of infinite Reynolds number, we re-examine the use of Lie point symmetries to reduce the latter PDE to a nonlinear ordinary differential equation (ODE) constituting a boundary value problem. Moreover, we present some physically interesting special cases of the model parameters for the boundary value problems. A short mathematical description of some transformations of variables, and the application of this method to finding analytical series solutions of these boundary value problems, is given in Section 3. Analysis of the so-called convergence-control parameter $C_{0}$, the scale coefficient $\lambda$ and the solutions obtained is given in Section 4. Finally, we show that the Loitsyansky integral invariant plays the role of a conservation law of a closure model for the von Kármán-Howarth equation in Appendix A.

\section{Basic equations}

2.1. The closed von Kármán-Howarth model equation In a pre-eminent paper, von Kármán and Howarth [9] derived a transport equation for the longitudinal twopoint velocity correlation in the limit of isotropic turbulence,

$$
\frac{\partial \overline{u^{\prime 2}(t)} f(r, t)}{\partial t}=\frac{1}{r^{4}} \frac{\partial}{\partial r}\left[r^{4}\left({\overline{u^{\prime 2}(t)}}^{3 / 2} h(r, t)+2 v \frac{\partial \overline{u^{\prime 2}(t)} f(r, t)}{\partial r}\right)\right] .
$$

Here $f$ and $h$ are respectively the normalized two-point double and triple velocity correlation, $\overline{u^{\prime 2}}$ is the root mean square value of the velocity fluctuations, $r$ is the correlation distance in correlation space, $t$ is time and $v$ is the kinematic viscosity. 
Oberlack [20] recognised that in the limit $v=0$ (or, equivalently, infinite Reynolds number) the unclosed equation (2.1) admits the two scaling groups

$$
\begin{array}{ll}
G^{a_{1}}: t^{*}=t, \quad r^{*}=e^{a_{1}} r, & {\overline{u^{\prime 2}}}^{*}=e^{2 a_{1}} \overline{u^{\prime 2}}, \quad f^{*}=f, \quad h^{*}=h, \\
G^{a_{2}}: t^{*}=e^{a_{2}} t, \quad r^{*}=r, & {\overline{u^{\prime 2}}}^{*}=e^{-2 a_{2}} \overline{u^{\prime 2}}, \quad f^{*}=f, \quad h^{*}=h,
\end{array}
$$

and a translation group in $t$,

$$
G^{a_{3}}: t^{*}=t+a_{3}, \quad r^{*}=r, \quad{\overline{u^{\prime 2}}}^{*}=\overline{u^{\prime 2}}, \quad f^{*}=f, \quad h^{*}=h,
$$

where $a_{1}, a_{2}, a_{3} \in \mathbb{R}$ are the group parameters. In infinitesimal form, (2.2) and (2.3) are given by

$$
X_{a_{1}}=r \frac{\partial}{\partial r}+2 \overline{u^{\prime 2}} \frac{\partial}{\partial \overline{u^{\prime 2}}}, \quad X_{a_{2}}=t \frac{\partial}{\partial t}-2 \overline{u^{\prime 2}} \frac{\partial}{\partial \overline{u^{\prime 2}}} .
$$

The groups $X_{a_{1}}, X_{a_{2}}$ may be combined to generate the two-parameter Lie scaling group

$$
G^{a_{1}, a_{2}}: t^{*}=e^{a_{2}} t, \quad r^{*}=e^{a_{1}} r, \quad{\overline{u^{\prime 2}}}^{*}=e^{2\left(a_{1}-a_{2}\right)} \overline{u^{\prime 2}}, \quad f^{*}=f, \quad h^{*}=h .
$$

Since (2.1) is an unclosed equation, Oberlack and Peters [21] developed a closure model connecting $f$ and $h$ which captures all properties of the Kolmogorov turbulence theory for $f$ and $h$ and also the proper limiting behaviour for $r \rightarrow 0$ and $r \rightarrow \infty$, namely:

- the Kolmogorov 2/3-law: $\overline{u^{\prime 2}}(1-f)=C(\varepsilon r)^{2 / 3}$ with the Kolmogorov constant $C \approx 2.3$ in the range $\eta \ll r \ll \ell_{t}$;

- the Kolmogorov 4/5-law: ${\overline{u^{\prime 2}}}^{3 / 2} h=2 \varepsilon r / 15$ in the range $\eta \ll r \ll \ell_{t}$ (the name "4/5-law" emerged from a somewhat different notation);

- as $r \rightarrow 0, \overline{u^{\prime 2}}(1-f)=\varepsilon r^{2} /(30 v)$ and $h=h_{0}^{\prime \prime \prime} r^{3} / 3$;

- as $r \rightarrow \infty, f$ decays faster than $r^{-2}$, where

$$
\eta=\left(\frac{v^{3}}{\varepsilon}\right)^{1 / 4} \text { and } \ell_{t}=\int_{0}^{\infty} f d r
$$

are the Kolmogorov and the integral length scales, respectively, and $\varepsilon$ is the dissipation rate of the turbulent kinetic energy.

Though not discussed by Oberlack and Peters [21], the closure model has to obey the full group properties of equation (2.1) and hence has to be conformal to (2.2)-(2.4). The model equation that actually captures all of these properties is given by

$$
{\overline{u^{\prime 2}}}^{3 / 2} h=2 \kappa_{2} r \sqrt{\overline{u^{\prime 2}}(1-f)} \frac{\partial \overline{u^{\prime 2}(t)} f(r, t)}{\partial r} \quad \text { with } \quad \kappa_{2}=\frac{\sqrt{2}}{5 \cdot C^{3 / 2}} .
$$

The resulting PDE, investigated below, is of nonlinear parabolic type:

$$
\frac{\partial \overline{u^{\prime 2}(t)} f(r, t)}{\partial t}=\frac{1}{r^{4}} \frac{\partial}{\partial r}\left[r^{4}\left(2 \kappa_{2} r \sqrt{\overline{u^{\prime 2}}(1-f)} \frac{\partial \overline{u^{\prime 2}(t)} f(r, t)}{\partial r}+2 v \frac{\partial \overline{u^{\prime 2}(t)} f(r, t)}{\partial r}\right)\right] .
$$


2.2. Group invariant solutions and emerging boundary value problems It was pointed out by Oberlack [20] that in the limit $v=0$, the two aforementioned scaling groups give rise to a family of self-similar solutions of the von Kármán-Howarth equation of the form

$$
\xi=\frac{r}{t^{2 /(\sigma+3)}}, \quad \hat{f}=\frac{\overline{u^{\prime 2}} f}{t^{-2(\sigma+1) /(\sigma+3)}}, \quad \hat{h}=\frac{{\overline{u^{\prime 2}}}^{3 / 2} h}{t^{-3(\sigma+1) /(\sigma+3)}}, \quad \sigma=\frac{2 a_{2}-3 a_{1}}{a_{1}},
$$

where $a_{1}, a_{2}$ are given above. These new variables may also be interpreted as invariants of the $G^{a_{1}, a_{2}}$ group. Here $r$ is scaled by the integral length scale $\ell_{t} \propto t^{2 /(\sigma+3)}$ and $\overline{u^{\prime 2}}$ accordingly by $\overline{u^{\prime 2}} \propto t^{-2(\sigma+1) /(\sigma+3)}$. Note that the translation group (2.4) may also be invoked, which gives a time shift $t \mapsto t+a$ to be employed later for completeness.

The invariants (2.6) enable us to reduce the number of variables in our closed model (2.5). As a result, we obtain the ODE

$$
\frac{2 \kappa_{2}}{\xi^{4}} \frac{d}{d \xi}\left[\xi^{5}(1-\hat{f})^{1 / 2} \frac{d \hat{f}}{d \xi}\right]+\delta \xi \frac{d \hat{f}}{d \xi}+\gamma \hat{f}=0
$$

subject to the boundary conditions

$$
\hat{f}(0)=1 \text { and } \hat{f}(\xi) \rightarrow 0 \text { as } \xi \rightarrow \infty,
$$

where

$$
\delta=\frac{2}{\sigma+3}, \quad \gamma=2 \frac{\sigma+1}{\sigma+3} .
$$

At this point $\sigma$ is undetermined and hence equation (2.7) together with (2.8) generates a one-parameter family of boundary value problems. We note also that in the limit of large Reynolds numbers, it is well known [24] that $\sigma$ is intimately related to the spatial decay of the normalized longitudinal correlation function $f$ or the low wave-number dependence of the energy spectrum. Further, we mention that $\kappa_{2}$ is not an additional branching parameter since it may for any $\kappa_{2} \neq 0$ always be absorbed into a rescaled $\xi$ without changing the boundary conditions (2.8).

The questions of which values are to be chosen for $\sigma$ and of the solvability of the corresponding nonlinear problem (2.7)-(2.8) are the subject of the present paper.

Interestingly enough, the ODE (2.7) displays no Lie point symmetry group no matter what value is chosen for $\sigma$. Still, using the theory of integrating factors [8], we find two values, $\sigma=0$ and $\sigma=4$, for which the ODE is integrable once. Note that here we have limited ourselves to the usual three cases where the integrating factors only depend on pairs of the set of variables $\xi, \hat{f}, d \hat{f} / d \xi$. These are essentially the only cases where the integrating factor may be algorithmically computed. The actual computations were conducted using the Maple package GeM [2].

First, taking $\sigma=0$, the integrating factor is a constant and we simply integrate equation (2.7) to obtain

$$
\kappa_{2}\left(3 \xi \sqrt{1-\hat{f}} \frac{d \hat{f}}{d \xi}-8(1-\hat{f})^{3 / 2}\right)+\xi \hat{f}=c_{1}^{\prime} .
$$


This equation may be transformed into an Abel equation which, for the present special case, is not further integrable. Here it is not considered further anyway since it corresponds to a nonphysical case, because $\overline{u^{\prime 2}}$ may not decay slower than $t^{-1}$.

However, it has already been shown by Oberlack [20] that, for the case $\sigma=4$, equation (2.7) admits the integrating factor $\xi^{4}$ and hence leads to

$$
7 \kappa_{2} \xi^{5}(1-\hat{f})^{1 / 2} \frac{d \hat{f}}{d \xi}+\xi^{5} \hat{f}=c_{1}^{\prime \prime} .
$$

Setting $c_{1}^{\prime \prime}=0$ allows for a final integration to

$$
\xi+c_{2}^{\prime \prime}=7 \kappa_{2}\left(\ln \left[\frac{1+(1-\hat{f})^{1 / 2}}{1-(1-\hat{f})^{1 / 2}}\right]-2(1-\hat{f})^{1 / 2}\right),
$$

the physical relevance of which is pointed out below. The boundary conditions (2.8) finally lead to $c_{2}^{\prime \prime}=0$. Considering $\sigma$ more from a physical point of view, we note that even though there is a vast literature on homogeneous isotropic turbulence in general, there are very few experimental results or results from direct numerical simulations of the Navier-Stokes equations devoted to the spatial decay of the longitudinal correlation function. This is in stark contrast to the well-known temporal decay according to a power law. According to similarity theory, they are closely coupled due to $\sigma$. Also, there is considerable uncertainty as to whether the asymptotic decay rates are unique for a given initial condition.

At this point it is relevant to consider the classical experiments of Stewart and Townsend [28]. Unfortunately we are not able to determine the value of $\sigma$ for large Reynolds number or the correlation distance $r$ from the measured values of $f$. It is well recognised that over the range of length scales available in the laboratory experiments, the decay rates of turbulent flows are affected by many factors.

This leads to the identification of conserved quantities which can be used to determine $\sigma$. Several invariants based on integrals of moments of the correlation function have been proposed for isotropic homogeneous turbulence. These include the Loitsyansky invariant [16] and the Birkhoff invariant [1]. Unfortunately neither of these invariants can be derived from first principles. In fact, they depend on initial conditions of forces responsible for creating grid turbulence.

The integral invariant

$$
\Lambda=\overline{u^{\prime 2}} \int_{0}^{\infty} r^{4} f(r, t) d r \quad \text { where } \Lambda \text { is a constant, }
$$

suggested by Loitsyansky [16] from an integrated form of the von Kármán-Howarth equation, is employed to determine the value of the parameter $\sigma$. For this we rewrite (2.11) invoking the invariants (2.6),

$$
\Lambda=\overline{u^{\prime 2}} \int_{0}^{\infty} r^{4} f(r, t) d r=t^{-2(\sigma+1) /(\sigma+3)} t^{10 /(\sigma+3)} \int_{0}^{\infty} \xi^{4} \hat{f}(\xi) d \xi,
$$

which determines $\sigma=4$ and hence $\gamma=10 / 7, \delta=2 / 7$. This leads to the only closedform solution (2.10) which defines a solution of (2.7)-(2.8) in implicit form. The 
computed evolution of $\overline{u^{\prime 2}}(t)$ and the integral length scale $\ell_{t}(t)$ satisfy

$$
\overline{u^{\prime 2}}(t) \propto(t+a)^{-10 / 7}, \quad \ell_{t} \propto(t+a)^{2 / 7}, \quad a \in \mathbb{R}
$$

and, not derived here, the low wave-number energy spectrum is $E(k)=\Lambda k^{4} /(3 \pi)$ [24].

Interestingly enough, the solution (2.10) with $c_{2}^{\prime \prime}=0$ coincides with the element of a Beltrami surface (or pseudo-sphere), a canonical surface of constant sectional curvature -1 . Details are given by Grebenev and Oberlack [6]. We only mention that in the new variables $x=\xi /\left(14 \kappa_{2}\right), y=\hat{f}^{1 / 2}$, equation (2.10) is transformed to the well-known tractrix equation [18]

$$
x=x(y)=-\left(a^{2}-y^{2}\right)^{1 / 2}+\frac{a}{2} \ln \left[\frac{a+\left(a^{2}-y^{2}\right)^{1 / 2}}{a-\left(a^{2}-y^{2}\right)^{1 / 2}}\right], \quad a=1,
$$

arising in differential geometry.

Birkhoff [1] suggested another invariant,

$$
B=\overline{u^{\prime 2}} \int_{0}^{\infty} r^{2}\left(3 f(r, t)+r \frac{\partial f}{\partial r}\right) d r \quad \text { where } B \text { is a constant. }
$$

By the same method as above, using the invariants of $G^{a_{1}, a_{2}}$, we find that $\sigma=2$. Therefore,

$$
\overline{u^{\prime 2}}(t) \propto(t+a)^{-6 / 5}, \quad \ell_{t} \propto(t+a)^{2 / 5},
$$

and the parameters of equation (2.7) are given by $\delta=2 / 5, \gamma=6 / 5$. The low wavenumber energy spectrum changes to $E(k)=B k^{2} / \pi$.

Finally, another less commonly known physical quantity which is an invariant is the constant integral length scale $\ell_{t}(t)$ proposed by Oberlack [20], which corresponds to high Reynolds number in turbulence in a box of constant length $L$. The two-parameter Lie scaling group $G^{a_{1}, a_{2}}$ is transformed into the one-parameter scaling group $G^{0, a_{2}}$. Here the integral length scale has reached the box size $\ell_{t}(t) \sim L$ which corresponds to $\sigma \rightarrow \infty$ and hence $\delta=0, \gamma=2$. This case may be explored by a direct numerical simulation in a given small computational box, running the computation sufficiently long that the integral length scale reaches the box size.

For different values of $\sigma$, we find the corresponding solutions of the boundary value problem (2.7)-(2.8) using the HAM [10,11] in the sections to follow.

\section{Generalized series solution}

3.1. Mathematical description The asymptotic behaviour of $\hat{f}(\xi)$ as $\xi \rightarrow \infty$ is considered in Section 4. Here we assume that $\hat{f} \sim 1-\alpha \xi^{\beta}$ as $\xi \rightarrow 0$. Substituting into equation (2.7), we obtain

$$
-2 \alpha^{3 / 2} \beta\left(4+\frac{3}{2} \beta\right) \xi^{3 \beta / 2-1}-\frac{\alpha \beta \delta}{k_{2}} \xi^{b}-\frac{a \gamma}{\kappa_{2}} \xi^{b}+\frac{\gamma}{\kappa_{2}}=0 .
$$


So $\alpha=\left(3 \gamma /\left(20 \kappa_{2}\right)\right)^{2 / 3}$ and $\beta=2 / 3$. Noting that equation (2.7) contains the nonpolynomial term $(1-\hat{f})^{1 / 2}$, we introduce the transformation

$$
\hat{f}=1-s^{2} \text {. }
$$

Then equation (2.7) changes to one of polynomial type,

$$
4 \xi s^{2} \frac{d^{2} s}{d \xi^{2}}+8 \xi s\left(\frac{d s}{d \xi}\right)^{2}+20 s^{2} \frac{d s}{d \xi}+2 \frac{\delta}{\kappa_{2}} \xi s \frac{d s}{d \xi}-\frac{\gamma}{\kappa_{2}}\left(1-s^{2}\right)=0,
$$

subject to the boundary conditions

$$
s(0)=0 \quad \text { and } \quad s(\xi) \rightarrow 1 \text { as } \xi \rightarrow \infty .
$$

By means of the transformation (3.2), the asymptotic behaviour as $\xi \rightarrow 0$ becomes

$$
s(\xi) \sim\left(\frac{3 \gamma}{20 \kappa_{2}}\right)^{1 / 3} \xi^{1 / 3}, \quad \xi \ll 1 .
$$

We further introduce the transformation

$$
\xi=y^{3}
$$

to achieve simple linear behaviour at the boundary $\xi \rightarrow 0$. The behaviour is fractional in $s(\xi)$, namely $s(\xi) \sim \xi^{1 / 3}$, from (3.1) and (3.2). With this transformation we obtain $s(y) \sim y$ for $y \rightarrow 0$. Equation (3.3) now becomes

$$
\frac{4}{9} y s^{2} \frac{d^{2} s}{d y^{2}}+\frac{52}{9} s^{2} \frac{d s}{d y}+\frac{8}{9} y s\left(\frac{d s}{d y}\right)^{2}+\frac{2}{3} \frac{\delta}{\kappa_{2}} y^{3} s \frac{d s}{d y}-\frac{\gamma}{\kappa_{2}} y^{2}\left(1-s^{2}\right)=0,
$$

subject to the boundary conditions

$$
s(0)=0 \quad \text { and } \quad s(y) \rightarrow 1 \text { as } y \rightarrow \infty .
$$

3.2. Homotopy analysis solution As pointed out by Liao [10], the essence of approximating a problem is to represent its solution by means of a complete set of base functions. Here we express $s(y)$ by the set of base functions

$$
\left\{(1+\lambda y)^{-m} \mid m \geq 0\right\}
$$

in the form

$$
s(y)=\sum_{m=0}^{\infty} c_{m}(1+\lambda y)^{-m},
$$

where the $c_{m}$ and the scale factor $\lambda$ are coefficients to be determined later. Since $y=\xi^{1 / 3}, s(y)=s(\xi) \rightarrow 1$ as $y \rightarrow \infty$. So it is reasonable to further express $s(y)$ as

$$
s(y)=1+\sum_{m=1}^{\infty} c_{m}\left\{(1+\lambda y)^{-m}\right\} .
$$

This so-called solution expression of $s(y)$ is useful for determining the other parameters. For simplicity, we introduce the transformation

$$
u=1+\lambda y \text {. }
$$


Then equation (3.4) becomes

$$
\begin{gathered}
\frac{4}{9}(u-1) \lambda s^{2} \frac{d^{2} s}{d u^{2}}+\frac{52}{9} \lambda s^{2} \frac{d s}{d u}+\frac{8}{9} \lambda(u-1) s\left(\frac{d s}{d u}\right)^{2} \\
+\frac{2}{3} \frac{\delta}{\kappa_{2}} \frac{(u-1)^{3}}{\lambda^{2}} s \frac{d s}{d u}-\frac{\gamma}{\kappa_{2}}\left(\frac{u-1}{\lambda}\right)^{2}\left(1-s^{2}\right)=0,
\end{gathered}
$$

subject to the boundary conditions

$$
s(1)=0 \quad \text { and } \quad s(u) \rightarrow 1 \text { as } u \rightarrow \infty .
$$

To satisfy the solution expression (3.5) and the boundary conditions (3.7), we choose the simple form

$$
s_{0}(u)=1-u^{-1}
$$

as the initial approximation of $s(u)$, and furthermore choose

$$
\mathcal{L}=u \frac{d f}{d u}+f
$$

as the auxiliary linear operator. The operator $\mathcal{L}$ has the properties

$$
\mathcal{L}\left[C u^{-1}\right]=0, \quad \mathcal{L}\left[u^{\beta}\right]=\frac{u^{\beta}}{\beta+1},
$$

where $C$ is constant, and $H(u)=u^{-3}$ is the auxiliary function chosen under the rule of coefficient ergodicity and solution expression (details are given by Liao [11, p. 19]).

According to the governing equation (3.6), we define the nonlinear operator

$$
\begin{aligned}
\mathcal{N}[\Phi(u ; q)]=\frac{4}{9} & (u-1) \lambda \Phi^{2}(u ; q) \frac{\partial^{2} \Phi(u ; q)}{\partial u^{2}}+\frac{52}{9} \lambda \Phi^{2}(u ; q) \frac{\partial \Phi(u ; q)}{\partial u} \\
& +\frac{8}{9} \lambda(u-1) \Phi(u ; q)\left(\frac{\partial \Phi(u ; q)}{\partial u}\right)^{2}+\frac{2}{3} \frac{\delta}{\kappa_{2}} \frac{(u-1)^{3}}{\lambda^{2}} \Phi(u ; q) \frac{\partial \Phi(u ; q)}{\partial u} \\
& -\frac{\gamma}{\kappa_{2}}\left(\frac{u-1}{\lambda}\right)^{2}\left(1-\Phi^{2}(u ; q)\right),
\end{aligned}
$$

from which we construct the so-called zeroth-order deformation equation

$$
(1-q) \mathcal{L}\left[\Phi(u ; q)-s_{0}(u)\right]=q C_{0} H(u) \mathcal{N}[\Phi(u ; q)],
$$

subject to the boundary conditions

$$
\Phi(1 ; q)=0, \quad \Phi(\infty ; q)=1,
$$

where $q \in[0,1]$ is called the embedding parameter and $C_{0} \neq 0$ the convergence-control parameter. When $q=0$,

$$
\Phi(u ; 0)=s_{0}(u) .
$$

When $q=1$, the zeroth-order deformation equations (3.9) and (3.10) are equivalent to the original equations (3.6) and (3.7), so that

$$
\Phi(u ; 1)=s(u) .
$$


Hence, as the embedding parameter $q$ increases from 0 to $1, \Phi(u ; q)$ varies (or deforms) from the initial approximation $s_{0}(u)$ to the solution $s(u)$ of (3.6) and (3.7).

Using Taylor's theorem and (3.11), we expand $\Phi(u ; q)$ in the power series

$$
\Phi(u ; q) \sim s_{0}(u)+\sum_{m=1}^{\infty} s_{m}(u) q^{m} \quad \text { where } s_{m}(u)=\left.\frac{1}{m !} \frac{\partial^{m} \Phi(u ; q)}{\partial q^{m}}\right|_{q=0} .
$$

Assuming that the convergence-control parameter $C_{0}$ is chosen such that the above series is convergent when $q=1$, we have, due to (3.12),

$$
s(u)=s_{0}(u)+\sum_{m=1}^{\infty} s_{m}(u)
$$

where $s_{m}(u), m=1,2,3, \ldots$, are obtained one by one from the linear high-order deformation equations (3.13) given below. The above expression gives a relationship between the initial guess $s_{0}(u)$ and the solution $s(u)$ of the original equations (3.6) and (3.7) via the unknown terms $s_{m}(u)$.

Differentiating $m$ times the zeroth-order deformation equations (3.9) and (3.10) with respect to the embedding parameter $q$, then setting $q=0$ and finally dividing by $m$ !, one obtains the so-called $m$ th-order deformation equation

$$
\mathcal{L}\left[s_{m}(u)-\chi_{m} s_{m-1}(u)\right]=C_{0} H(u) R_{m}(u),
$$

subject to the boundary conditions

$$
s_{m}(1)=0 \quad \text { and } \quad s_{m}(\infty)=0,
$$

where

$$
\begin{aligned}
R_{m}(u)=\frac{4}{9}( & u-1) \lambda \sum_{j=0}^{m-1} X_{j}(u) s_{m-1-j}^{\prime \prime}(u)+\frac{52}{9} \lambda \sum_{j=0}^{m-1} X_{j}(u) s_{m-1-j}^{\prime}(u) \\
& +\frac{8}{9} \lambda(u-1) \sum_{j=0}^{m-1} Y_{j}(u) s_{m-1-j}^{\prime}(u)+\frac{2}{3} \frac{\delta}{\kappa_{2}} \frac{(u-1)^{3}}{\lambda^{2}} Y_{m-1}(u) \\
& -\frac{\gamma}{\kappa_{2}}\left(\frac{u-1}{\lambda}\right)^{2}\left(1-\chi_{m}-X_{m-1}(u)\right),
\end{aligned}
$$

with the definitions

$$
X_{k}(u)=\sum_{i=0}^{k} s_{i}(u) s_{k-i}(u), \quad Y_{k}(u)=\sum_{i=0}^{k} s_{i}(u) s_{k-i}^{\prime}(u), \quad \chi_{m}= \begin{cases}0 & \text { when } m \leq 1 \\ 1 & \text { when } m>1\end{cases}
$$

Here the prime denotes the derivative with respect to $u$. The solution of equation (3.13) can be expressed as

$$
s_{m}(u)=s^{*}(u)+C u^{-1},
$$


where $C$ is an integral constant and $s^{*}(u)$ is a special solution of equation (3.13). The constant $C$ is determined by the boundary condition (3.14) at $u=1$. We find that

$$
s_{m}(u)=\sum_{n=0}^{7 m+1} \frac{\alpha_{m, n}}{u^{n}},
$$

where the $\alpha_{m, n}$ are constant coefficients. Substituting the above expression into the $m$ th-order deformation equations (3.13) and (3.14) yields the recurrence formulas

$$
\begin{aligned}
\alpha_{m, n}=\chi_{m} & \chi_{7 m-4-n} \alpha_{m-1, n}+\frac{C_{0}}{n-1}\left\{\frac{4}{9} \lambda\left(\Psi_{m, n-1}^{1}-\Psi_{m, n}^{1}\right)+\frac{52}{9} \lambda \Psi_{m, n}^{2}\right. \\
& -\frac{8}{9} \lambda\left(\Psi_{m, n}^{3}-\Psi_{m, n-1}^{3}\right)-\frac{2 \delta}{3 \kappa_{2} \lambda^{2}}\left(\Pi_{m, n-3}^{2}-3 \Pi_{m, n-2}^{2}+3 \Pi_{m, n-1}^{2}-\Pi_{m, n}^{2}\right) \\
& +\frac{\gamma}{\kappa_{2} \lambda^{2}}\left(1-\chi_{m}\right)(n-1)\left(\frac{\chi_{n-1} \chi_{5-n}}{2}-2 \chi_{n} \chi_{4-n}\right)-\frac{\gamma}{\kappa_{2} \lambda^{2}} \chi_{7 m-2-n} \chi_{n} \Gamma_{m-1, n-1}^{1} \\
& \left.+\frac{\gamma}{\kappa_{2} \lambda^{2}}\left(2 \Pi_{m, n}^{1}-\Pi_{m, n-1}^{1}\right)\right\}, \quad m \geq 1, n \geq 2,
\end{aligned}
$$

where

$$
\begin{aligned}
& \Psi_{m, n}^{1}=\chi_{7 m+2-n} \chi_{n-3} \Delta_{m, n-4}^{1}, \\
& \Psi_{m, n}^{2}=\chi_{7 m+2-n} \chi_{n-3} \Delta_{m, n-4}^{2}, \\
& \Psi_{m, n}^{3}=\chi_{7 m+2-n} \chi_{n-4} \Delta_{m, n-4}^{3}, \\
& \Pi_{m, n}^{1}=\chi_{7 m-1-n} \chi_{n} \Gamma_{m-1, n-2}^{1}, \\
& \Pi_{m, n}^{2}=\chi_{7 m-2-n} \chi_{n} \Gamma_{m-1, n-1}^{2},
\end{aligned}
$$

and

$$
\begin{aligned}
\Gamma_{j, r}^{1} & =\sum_{i=0}^{j} \sum_{n=\max \{0, r-7 j+7 i-1\}}^{\min \{7 i+1, r\}} \alpha_{i, n} \alpha_{j-i, r-n}, \\
\Gamma_{j, r}^{2} & =\sum_{i=0}^{j} \sum_{n=\max \{0, r-7 j+7 i-1\}}^{\min \{7 i+1, r-1\}}(r-n) \alpha_{i, n} \alpha_{j-i, r-n}, \\
\Delta_{m, t}^{1}= & \sum_{j=0}^{m-1} \sum_{r=\max \{0, t-7 m+7 j+6\}}^{\min \{7 j+2, t-1\}}(t-r)(t-r+1) \alpha_{m-1-j, t-r} \Gamma_{j, r}^{1}, \\
\Delta_{m, t}^{2}= & \sum_{j=0}^{m-1} \sum_{\min \{7 j+2, t-1\}}(t-r) \alpha_{m-1-j, t-r} \Gamma_{j, r}^{1}, \\
\Delta_{m, t}^{3}= & \sum_{j=0}^{m-1} \sum_{\min \{0, t-7 m+7 j+6\}} \sum_{r=\max \{1, t-7 m+7, t-1\}}(t-r) \alpha_{m-1-j, t-r} \Gamma_{j, r}^{2} .
\end{aligned}
$$


Moreover,

$$
\alpha_{m, 1}=-\sum_{n=2}^{7 m+1} \alpha_{m, n}, \quad m \geq 1 .
$$

Due to the definition (3.8) of $s_{0}(u)$,

$$
\alpha_{0,0}=1, \quad \alpha_{0,1}=-1 .
$$

From the solution expressions (3.5),

$$
\alpha_{m, 0}=0 \text { for } m \geq 1 \text {. }
$$

Using the recurrence formulas (3.15)-(3.26) and the known coefficients given by (3.27) and (3.28), all other coefficients $\alpha_{m, n}$ can be calculated successively. This provides us with an explicit series solution of equation (3.6):

$$
s(u)=\sum_{m=0}^{\infty} \sum_{n=0}^{7 m+1} \frac{\alpha_{m, n}}{u^{n}} .
$$

The corresponding $k$ th-order approximation reads

$$
s(u) \approx \sum_{m=0}^{k} \sum_{n=0}^{7 m+1} \frac{\alpha_{m, n}}{u^{n}} .
$$

Therefore, we have an explicit, fully analytical solution of equation (2.7):

$$
\hat{f}(\xi) \approx 1-\left(\sum_{m=0}^{k} \sum_{n=0}^{7 m+1} \frac{\alpha_{m, n}}{\left(1+\lambda \xi^{1 / 3}\right)^{n}}\right)^{2} .
$$

\section{Result analysis}

Our series solution (3.29) for $\hat{f}(\xi)$ explicitly contains the convergence-control parameter $C_{0}$ and scale coefficient $\lambda$. The convergence-control parameter $C_{0}$ is employed to control the convergence of approximations and adjust convergence regions. It is the introduction of $C_{0}$ that greatly improves the early homotopy analysis method [12]. Liao [13] proved that the famous Euler transform is equivalent to the homotopy analysis method for some special choices of the initial guess and the auxiliary linear operator in the case of the simplest deformation functions $A(p)=$ $B(p)=p$ and when there is only one auxiliary parameter $C_{0}$. This fact explains why the convergence of solution series given by the homotopy analysis method can be guaranteed. If the convergence-control parameter $C_{0}$ and scale coefficient $\lambda$ are properly chosen, the homotopy-series solution will converge quickly. So we try to find the best values for them.

Following Liao [14], we use the average residual error

$$
E_{k}=\frac{1}{K} \sum_{j=0}^{K}[\mathcal{N}(\hat{f}(j \Delta \xi))]^{2}
$$


TABLE 1. The values of $C_{0}$ and $\lambda$ for the 15 th-order homotopy-series solution.

\begin{tabular}{llllll}
\hline \multirow{2}{*}{$C_{0}$} & \multicolumn{2}{c}{$\sigma=4$} & & \multicolumn{2}{c}{$\sigma=2$} \\
\cline { 2 - 3 } \cline { 5 - 6 } & $\lambda$ & $E_{15}$ & & $E_{15}$ \\
\hline-0.9 & 1.95 & 0.108685 & & 1.83 & 0.0419714 \\
-1.0 & 2.04 & 0.0206401 & & 1.92 & 0.00602734 \\
-1.1 & 2.12 & 0.0186465 & & 2.00 & 0.00267042 \\
-1.2 & 2.19 & 0.0342259 & & 2.08 & 0.00551670 \\
-1.3 & 2.26 & 0.0490727 & & 2.16 & 0.00848203 \\
-1.4 & 2.33 & 0.0611937 & & 2.23 & 0.0102360 \\
-1.5 & 2.41 & 0.0688688 & & 2.31 & 0.0107048 \\
\hline
\end{tabular}

TABLE 2. Comparison of the homotopy-series solution and homotopy-Padé solution given by $\sigma=4$, $C_{0}=-1.1, \lambda=2.12$ with the results of Grebenev and Oberlack [6].

\begin{tabular}{llllll}
\hline$\xi$ & $\begin{array}{l}\text { 50th-order } \\
\text { homotopy-series }\end{array}$ & $\begin{array}{l}\text { 60th-order } \\
\text { homotopy-series }\end{array}$ & $\begin{array}{l}\text { Order [20,20] } \\
\text { homotopy-Padé }\end{array}$ & $\begin{array}{l}\text { Order [30,30] } \\
\text { homotopy-Padé }\end{array}$ & $\begin{array}{l}\text { Grebenev and } \\
\text { Oberlack }\end{array}$ \\
\hline 0.5 & 0.2393 & 0.2393 & 0.2393 & 0.2393 & 0.2393 \\
1.0 & 0.08631 & 0.08631 & 0.08631 & 0.08631 & 0.08631 \\
1.5 & 0.03282 & 0.03282 & 0.03282 & 0.03282 & 0.03282 \\
2.0 & 0.01269 & 0.01269 & 0.01269 & 0.01269 & 0.01269 \\
2.5 & $4.941 \times 10^{-3}$ & $4.940 \times 10^{-3}$ & $4.939 \times 10^{-3}$ & $4.940 \times 10^{-3}$ & $4.940 \times 10^{-3}$ \\
3.0 & $1.928 \times 10^{-3}$ & $1.927 \times 10^{-3}$ & $1.922 \times 10^{-3}$ & $1.927 \times 10^{-3}$ & $1.927 \times 10^{-3}$ \\
3.5 & $7.537 \times 10^{-4}$ & $7.524 \times 10^{-4}$ & $7.526 \times 10^{-4}$ & $7.524 \times 10^{-4}$ & $7.524 \times 10^{-4}$ \\
4.0 & $2.978 \times 10^{-4}$ & $2.943 \times 10^{-4}$ & $2.941 \times 10^{-4}$ & $2.939 \times 10^{-4}$ & $2.939 \times 10^{-4}$ \\
4.5 & $1.217 \times 10^{-4}$ & $1.160 \times 10^{-4}$ & $1.147 \times 10^{-4}$ & $1.148 \times 10^{-4}$ & $1.148 \times 10^{-4}$ \\
5.0 & $5.273 \times 10^{-5}$ & $4.639 \times 10^{-5}$ & $4.489 \times 10^{-5}$ & $4.486 \times 10^{-5}$ & $4.485 \times 10^{-5}$ \\
\hline
\end{tabular}

of equation (3.29) versus $\lambda$ to determine the value of $\lambda$ after assigning a group value of $C_{0}$. Here we set $k=15, \Delta \xi=10 / K$ and $K=40$. The relationship between $C_{0}, \lambda$ and $E_{15}$ is shown in Table 1 for $\sigma=4$ and $\sigma=2$. For $\sigma=4$, selecting $C_{0}=-1.1$ and $\lambda=2.12$, we find that the homotopy-series solution agrees well with the solution given by Grebenev and Oberlack [6], as shown in Table 2. Notice that as $\xi$ becomes larger, the homotopy-Padé technique, the details of which are given by Liao [11, p. 38], can give a much better solution. Indeed, the homotopy-Padé technique can increase the accuracy and convergence of the solution [15]. When $\sigma=2$, the optimal values of $C_{0}$ and $\lambda$ are -1.1 and 2 , respectively. The comparison between homotopy-series and homotopy-Padé solutions is shown in Table 3.

It is surprising that we can also find homotopy-series solutions for other values of $\sigma$, for example, 1 and 3, for which there is no physical meaning. Having attempted hundreds of combinations of values of $\sigma, C_{0}$ and $\lambda$, we could obtain explicit, fully analytical solutions of (3.6) for $-1<\sigma \leq 4$. However, in order to consider viscous 
TABLE 3. Comparison between homotopy-series and homotopy-Padé solutions: $\sigma=2, C_{0}=-1.1, \lambda=2$.

\begin{tabular}{lllll}
\hline$\xi$ & $\begin{array}{l}\text { 50th-order } \\
\text { homotopy-series }\end{array}$ & $\begin{array}{l}\text { 60th-order } \\
\text { homotopy-series }\end{array}$ & $\begin{array}{l}\text { Order [20,20] } \\
\text { homotopy-Padé }\end{array}$ & $\begin{array}{l}\text { Order [30,30] } \\
\text { homotopy-Padé }\end{array}$ \\
\hline 0.5 & 0.3025 & 0.3025 & 0.3025 & 0.3025 \\
1.0 & 0.1429 & 0.1429 & 0.1429 & 0.1429 \\
1.5 & 0.07601 & 0.07601 & 0.07601 & 0.07601 \\
2.0 & 0.04392 & 0.04392 & 0.04392 & 0.04392 \\
2.5 & 0.02711 & 0.02711 & 0.02711 & 0.02711 \\
3.0 & 0.01768 & 0.01768 & 0.01768 & 0.01768 \\
3.5 & 0.01208 & 0.01208 & 0.01208 & 0.01208 \\
4.0 & $8.576 \times 10^{-3}$ & $8.575 \times 10^{-3}$ & $8.575 \times 10^{-3}$ & $8.575 \times 10^{-3}$ \\
4.5 & $6.290 \times 10^{-3}$ & $6.289 \times 10^{-3}$ & $6.289 \times 10^{-3}$ & $6.289 \times 10^{-3}$ \\
5.0 & $4.742 \times 10^{-3}$ & $4.740 \times 10^{-3}$ & $4.740 \times 10^{-3}$ & $4.740 \times 10^{-3}$ \\
\hline
\end{tabular}

TABLe 4. Order [30, 30] homotopy-Padé solution for $\sigma=1,3$.

\begin{tabular}{llllll}
\hline$\xi$ & $\sigma=1$ & $\sigma=3$ & $\xi$ & $\sigma=1$ & $\sigma=3$ \\
\hline 0.5 & 0.3669 & 0.2644 & 3.0 & 0.04950 & $6.434 \times 10^{-3}$ \\
1.0 & 0.2070 & 0.1079 & 3.5 & 0.03864 & $3.685 \times 10^{-3}$ \\
1.5 & 0.1317 & 0.04848 & 4.0 & 0.03096 & $2.225 \times 10^{-3}$ \\
2.0 & 0.09036 & 0.02329 & 4.5 & 0.02535 & $1.409 \times 10^{-3}$ \\
2.5 & 0.06550 & 0.01189 & 5.0 & 0.02112 & $9.305 \times 10^{-4}$ \\
\hline
\end{tabular}

effects, we focus on $1 \leq \sigma \leq 4$ in this paper. The values of $C_{0}$ and $\lambda$ are determined by

$$
\begin{gathered}
C_{0}=-\frac{3}{2}+\frac{13}{30} \sigma-\frac{3}{20} \sigma^{2}+\frac{1}{60} \sigma^{3}, \\
\lambda=2-\frac{29}{300} \sigma+\frac{13}{200} \sigma^{2}-\frac{1}{120} \sigma^{3} .
\end{gathered}
$$

For $\sigma=1,3$, the order [30, 30] homotopy-Padé solutions are shown in Table 4. We also give 20th-order homotopy-series solutions of the normalized longitudinal two-point double velocity correlation function $\hat{f}(\xi)$ and transversal two-point double velocity correlation function $\hat{g}(\xi)$ for $\sigma=1,2,3,4$ in Figure 1. Here

$$
\hat{g}(\xi)=\hat{f}(\xi)+\frac{\xi}{2} \frac{d \hat{f}(\xi)}{d \xi} .
$$

These two solutions are characterized by the following properties. The function $\hat{f}(\xi)$ is positive everywhere and asymptotically tends to zero. For $\sigma=1, \hat{g}(\xi)$ is always positive and asymptotically tends to zero. For $\sigma=2,3,4, \hat{g}(\xi)$ is positive for $\xi \leq \xi^{*}$, and after the point $\xi^{*}$, where $\hat{g}\left(\xi^{*}\right)=0$, it is negative everywhere and tends to zero as $\xi \rightarrow \infty$. By continuity, there exists a $\sigma^{*}$ between 1 and 2 corresponding to $\hat{g}\left(\xi^{*}\right)$ where $\xi^{*}=\infty$. 


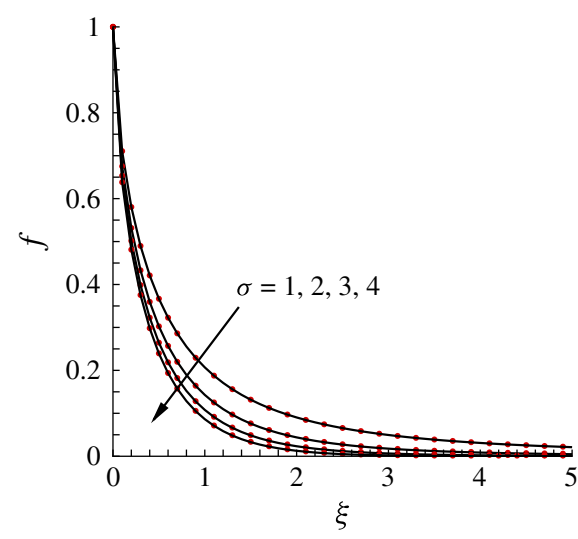

(a) $\hat{f}(\xi)$

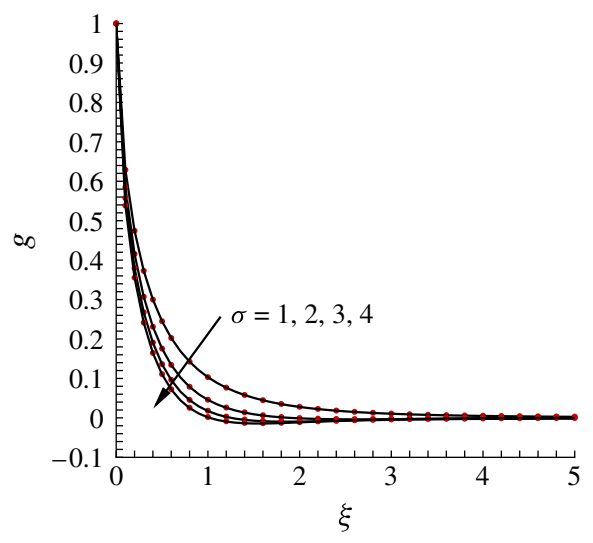

(b) $\hat{g}(\xi)$

FIgURE 1. Homotopy solution of (a) $\hat{f}(\xi)$ and (b) $\hat{g}(\xi)$. Solid line: order $[30,30]$ homotopy-Padé approximation. Dots: 20th-order HAM approximation.

In addition, Figure 1 shows that as $\sigma$ changes from 4 to 1 , both $\hat{f}(\xi)$ and $\hat{g}(\xi)$ decay more and more slowly. This suggests that $\hat{f}(\xi)$ and $\hat{g}(\xi)$ may first exponentially decrease in the case $\sigma=4$ and then algebraically tend to zero for $\sigma=3,2,1$. Figure 2 shows that the HAM solutions agree well with the asymptotic expressions discussed below.

Considering the asymptotic behaviour of $\hat{f}$ for $\xi \rightarrow \infty$, we use the property that $(1-\hat{f})^{1 / 2} \rightarrow 1$ as $\xi \rightarrow \infty$. Therefore, formally the equation

$$
\frac{2 \kappa_{2}}{\xi^{4}} \frac{d}{d \xi}\left[\xi^{5} \frac{d \hat{f}}{d \xi}\right]+\delta \xi \frac{d \hat{f}}{d \xi}+\gamma \hat{f}=0
$$

is obtained from (2.7) in the limiting case $\xi \rightarrow \infty$. In order to study the asymptotic behaviour of solutions of equation (4.1), we make some simple transformations. Let us divide this equation by $\delta$ and introduce the rescaled variable $\breve{\xi}=2\left(\left(\delta / 2 \kappa_{2}\right) \xi\right)^{1 / 2}$. Thus, we rewrite this equation in the form

$$
\frac{1}{\breve{\xi}^{9}} \frac{d}{d \breve{\xi}}\left[\breve{\xi}^{9} \frac{d \breve{f}}{d \breve{\xi}}\right]+\frac{\breve{\xi}}{2} \frac{d \breve{f}}{d \breve{\xi}}+\frac{\gamma}{\delta} \breve{f}=0, \quad \breve{f}(\breve{\xi})=\hat{f}(\xi)
$$

or

$$
\frac{d^{2} \breve{f}}{d \breve{\xi}^{2}}+\left(\frac{9}{\breve{\xi}}+\frac{\breve{\xi}}{2}\right) \frac{d \breve{f}}{d \breve{\xi}}+(\sigma+1) \breve{f}=0
$$

using the formulas (2.9) for $\delta$ and $\gamma$. Oberlack and Peters [22, p. 89] proved (see also Erdélyi [4, p. 302]) that for $\sigma \in(1,4)$ this equation admits decreasing solutions of the asymptotic form

$$
\breve{f}(\breve{\xi})=M_{1} \breve{\xi}^{-2(\sigma+1)}+M_{2} \breve{\xi}^{2(\sigma-4)} e^{-\breve{\xi}^{2} / 4} \text { for } \breve{\xi} \rightarrow \infty
$$




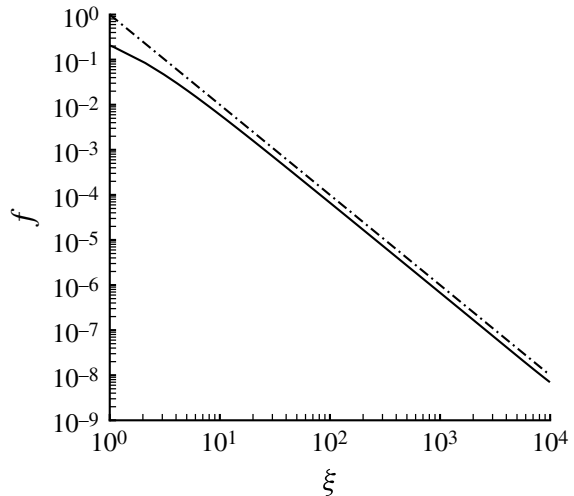

(a) $\sigma=1$

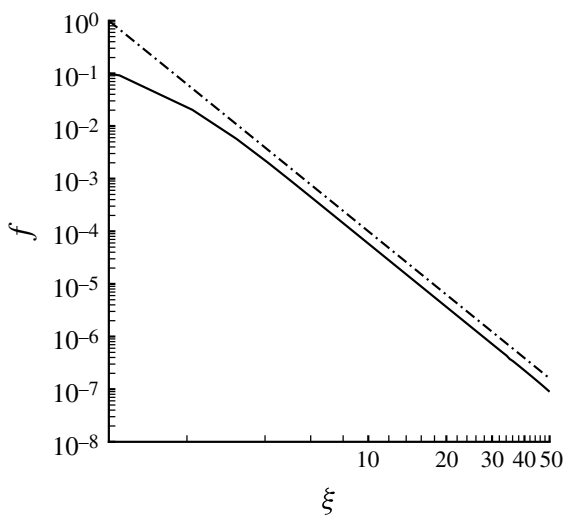

(c) $\sigma=3$

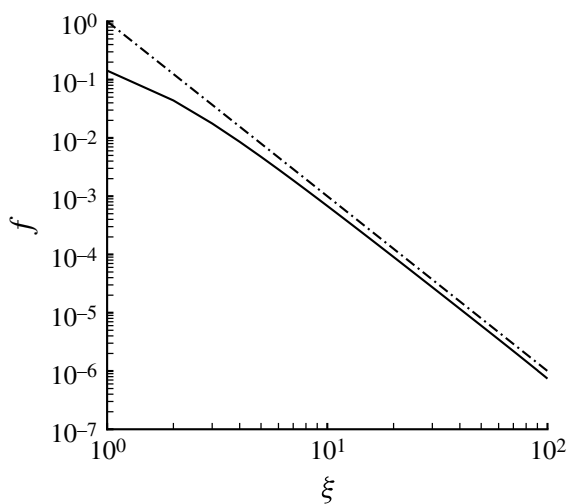

(b) $\sigma=2$

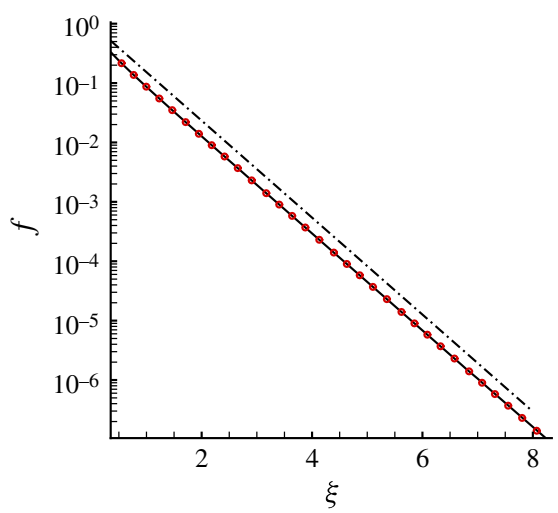

(d) $\sigma=4$

FIGURE 2. Asymptotic behaviour of $\hat{f}(\xi)$ as $\xi \rightarrow \infty$. Solid lines show (a), (b), (d) order [30, 30] and (c) order $[35,35]$ homotopy-Padé approximations. Dash-dotted lines are asymptotics given by (a), (b), (c) equation (4.2) and (d) equation (4.3). For (d), dots indicate the exact solution given by (2.10).

where $M_{1}, M_{2}>0$ are constants. Interestingly enough, the solution in the previous section shows that for $\sigma<4$ the algebraic part is dominant for $\breve{\xi} \rightarrow \infty$. However, if $\sigma=4$ then

$$
\breve{f}(\breve{\xi})=M e^{-\breve{\xi}^{2} / 4},
$$

and for $\sigma>4$ the equation has no everywhere-positive solutions. In the case $\sigma=4$, we construct the explicit deformation of the exact solution

$$
\xi=7 \kappa_{2}\left(\ln \left[\frac{1+(1-\hat{f})^{1 / 2}}{1-(1-\hat{f})^{1 / 2}}\right]-2(1-\hat{f})^{1 / 2}\right)
$$

of the original equation (2.7) into the solution

$$
\hat{f}=\exp \left(-\frac{\xi}{7 \kappa_{2}}\right)
$$


of (4.1). Recall that these functions are subject to the boundary conditions (2.8). Now we consider the problem

$$
\begin{gathered}
7 \kappa_{2}\left(1-\varepsilon \hat{f}_{\varepsilon}\right)^{1 / 2} \frac{d \hat{f}_{\varepsilon}}{d \xi}+\hat{f}_{\varepsilon}=0, \\
\hat{f}_{\varepsilon}(0)=1, \quad 0 \leq \varepsilon \leq 1,
\end{gathered}
$$

and notice that for $\varepsilon=1$ and $\varepsilon=0$ the solutions $\hat{f}_{1}$ and $\hat{f}_{0}$ of the problem (4.6)-(4.7) coincide with the functions defined by the formulas (4.4) and (4.5), respectively. Instead of $f_{\varepsilon}$, we introduce the function $G_{\varepsilon}=\varepsilon \hat{f}_{\varepsilon}$. A differential equation for $G_{\varepsilon}$ is

$$
\frac{d G_{\varepsilon}}{d \xi}=-\frac{G_{\varepsilon}}{7 \kappa_{2}\left(1-G_{\varepsilon}\right)^{1 / 2}}, \quad \xi \in(0, \infty) .
$$

Here $G_{\varepsilon}$ satisfies the initial condition

$$
G_{m}(0)=\varepsilon_{m}, \quad 0<\varepsilon_{m}<1 .
$$

The solution of the problem (4.8)-(4.9) reads

$$
\begin{aligned}
\frac{1}{14 \kappa_{2}} \xi=-( & \left.1-G_{\varepsilon}\right)^{1 / 2}+(1-\varepsilon)^{1 / 2} \\
& +\frac{1}{2} \ln \left[\frac{1+\left(1-G_{\varepsilon}\right)^{1 / 2}}{1-\left(1-G_{\varepsilon}\right)^{1 / 2}}\right]-\frac{1}{2} \ln \left[\frac{1+(1-\varepsilon)^{1 / 2}}{1-(1-\varepsilon)^{1 / 2}}\right] .
\end{aligned}
$$

It is a positive monotonically decreasing function as $\xi \rightarrow \infty$ which achieves the maximum value $\varepsilon$ at $\xi=0$. We multiply (4.10) by -2 and rewrite it in the form

$$
\begin{aligned}
\exp \left(-\frac{\xi}{7 \kappa_{2}}\right)= & \left.\frac{G_{\varepsilon}}{(1}+\left(1-G_{\varepsilon}\right)^{1 / 2}\right)^{2} \\
& \times \exp \left(2\left(1-G_{\varepsilon}\right)^{1 / 2} \frac{\left(1-(1-\varepsilon)^{1 / 2}\right)^{2}}{\varepsilon}\right) \exp \left(-2(1-\varepsilon)^{1 / 2}\right) .
\end{aligned}
$$

For small values of $\varepsilon$, and hence small values of $G_{\varepsilon}$,

$$
\begin{aligned}
& \exp \left(-\frac{\xi}{7 \kappa_{2}}\right)=\frac{G_{\varepsilon}}{\left(1+\left(1-G_{\varepsilon}\right)^{1 / 2}\right)^{2}}\left(1+\left(1-G_{\varepsilon}\right)^{1 / 2}+o\left(G_{\varepsilon}\right)\right)^{2} \\
& \times \frac{\left(1-(1-\varepsilon)^{1 / 2}\right)^{2}}{\varepsilon}\left(1-(1-\varepsilon)^{1 / 2}+o(\varepsilon)\right)^{2} .
\end{aligned}
$$

From (4.11) it follows that for small values of $\varepsilon$,

$$
\hat{f_{\varepsilon}}=\exp \left(-\frac{\xi}{7 \kappa_{2}}\right)+o(\varepsilon)
$$

uniformly in $\xi$. Hence $\hat{f}_{1}(\xi)$ is deformed continuously into the function $\hat{f}_{0}(\xi)$ as $\varepsilon \rightarrow 0$. This reflects the well-known property about continuous dependence on the initial data of solutions of differential equations. Geometrically, this deformation corresponds to a shift of the graph of $\hat{f}_{1}(\xi)$ along the $\xi$-axis by a quantity $a_{\varepsilon}$ such that $a_{\varepsilon} \rightarrow-\infty$ as 
$\varepsilon \rightarrow 0$. We note that the explicit integration of equations (2.7) and (4.1) is associated with the Loitsyansky integral invariant $\Lambda$ (see (2.11)). The explicit solution (4.5) obtained for $\sigma=4$ demonstrates that $\Lambda<\infty$ and, as a consequence of the above asymptotic analysis, the same property holds for the solution (4.4) of the original equation. In Appendix A, we prove that the Loitsyansky integral invariant indeed plays the role of a conservation law for the evolution equation (2.5).

\section{Conclusion}

In this paper we have applied the HAM to deal with the von Kármán-Howarth equation (2.7) associated with the boundary conditions (2.8). Our results show that the HAM solutions agree well with the asymptotic expressions

$$
\hat{f}(\xi)=N_{1} \xi^{-(\sigma+1)}+N_{2} \xi^{\sigma-4} e^{-\delta \xi /\left(2 \kappa_{2}\right)} \quad \text { as } \xi \rightarrow \infty
$$

for $1 \leq \sigma \leq 4$, where $N_{1}, N_{2}>0$ are constants. The values $\sigma=2,4$ correspond to the classical invariants of Birkhoff and Loitsyansky, and with the present series solutions derived by the HAM we have obtained an explicit solution of the von KármánHowarth equation and the relationship between temporal and spatial decay of the longitudinal correlation function.

This result shows that there is no unique power-law behaviour for decaying turbulence but that instead it strongly depends on the initial distribution of the correlation function. This, however, is only true for solutions for freely decaying turbulence obtained by solving the Navier-Stokes equations. Only very recently, an exponential decay of turbulence was observed which was obtained by solving related fractal equations [7, 27]. This rather unusual scaling behaviour was later unravelled and traced back to a set of new symmetries which only originate in the multi-point equation and hence are named statistical symmetries $[23,26]$ since they do not have a counterpart in the Euler or Navier-Stokes equations.

\section{Acknowledgement}

The first and last authors thank the State Key Lab of Ocean Engineering for partial financial support (Approval No. GKZD010029).

\section{Appendix A. The Loitsyansky invariant}

The main aim of this section is to prove that the Loitsyansky invariant $\Lambda$ is a conservation law of the problem below under the assumption that $\Lambda<\infty$ at the initial time. In order to establish this assertion, we have to show that solutions of the problem are rapidly decreasing functions as $r \rightarrow \infty$, uniformly in $t$. Direct calculation of the time derivative of $\Lambda(t)$ using the corresponding governing differential equation shows that $d \Lambda / d t \equiv 0$ if $\partial B_{L L}(r, t) / \partial r$ decays faster than $r^{-5}$ as $r \rightarrow \infty$. In other words, we have to prove that $B_{L L}(r, \cdot)$ belongs to a space of sufficiently rapidly decreasing 
functions. We prove this using the semigroup approach and, as a result, we construct a semigroup on a suitable function space which admits the Loitsyansky invariant.

We consider the von Kármán-Howarth equation (2.5). The key is that according to the asymptotic property that $(1-f)^{1 / 2} \rightarrow 1$ for large values of $r$,

$$
\frac{\partial \overline{u^{\prime 2}(t)} f(r, t)}{\partial t}=\frac{1}{r^{4}} \frac{\partial}{\partial r}\left[r^{4}\left(2 \kappa_{2} r \sqrt{\overline{u^{\prime 2}}} \frac{\partial \overline{u^{\prime 2}(t)} f(r, t)}{\partial r}+2 v \frac{\partial \overline{u^{\prime 2}(t)} f(r, t)}{\partial r}\right)\right],
$$

which describes the asymptotic behaviour of solutions to (2.5). In order to study equation (A.1), we rewrite it in the form

$$
\frac{\partial B_{L L}}{\partial t}=2 \kappa_{2} \sqrt{\overline{u^{\prime 2}}(t)}\left(r \frac{\partial^{2}}{\partial r^{2}} B_{L L}+5 \frac{\partial}{\partial r} B_{L L}\right)+2 v\left(\frac{\partial^{2}}{\partial r^{2}} B_{L L}+\frac{4}{r} \frac{\partial}{\partial r} B_{L L}\right),
$$

using the classical definition $B_{L L}(r, t)=\overline{u^{\prime 2}(t)} f(r, t)$ for the longitudinal correlation function $B_{L L}(r, t)$. Equation (A.2) is supplemented by the initial-boundary conditions

$$
\begin{gathered}
B_{L L}(r, 0)=B_{0 L L}(r), \quad r \geq 0, \\
B_{L L, L}=2 \kappa_{2} r \sqrt{\overline{u^{\prime 2}}(t)} \frac{\partial B_{L L}}{\partial r}=0, \quad r=0, B_{L L}=0, r \rightarrow \infty .
\end{gathered}
$$

Instead of problem (A.1)-(A.4), we study two individual problems which lead to solving (A.1)-(A.4):

$$
\frac{\partial B_{1 L L}}{\partial t}=2 \kappa_{2} \sqrt{\overline{u^{\prime 2}}(t)}\left(r \frac{\partial^{2}}{\partial r^{2}} B_{1 L L}+5 \frac{\partial}{\partial r} B_{1 L L}\right)
$$

and

$$
\frac{\partial B_{2 L L}}{\partial t}=2 v\left(\frac{\partial^{2}}{\partial r^{2}} B_{2 L L}+\frac{4}{r} \frac{\partial}{\partial r} B_{2 L L}\right)
$$

subject to

$$
\begin{gathered}
B_{i L L}(r, 0)=B_{i 0 L L}(r), \quad r \geq 0, i=1,2, \\
2 \kappa_{2} r \sqrt{\overline{u^{\prime 2}}(t)} \frac{\partial B_{i L L}}{\partial r}=0, \quad r=0, B_{i L L}=0, r \rightarrow \infty .
\end{gathered}
$$

Let us consider the operator (the right-hand side of (A.2))

$$
H=2 \kappa_{2} \sqrt{\overline{u^{\prime 2}}(t)}\left(r \frac{\partial^{2}}{\partial r^{2}}+5 \frac{\partial}{\partial r}\right)+2 v\left(\frac{\partial^{2}}{\partial r^{2}}+\frac{4}{r} \frac{\partial}{\partial r}\right),
$$

which can be rewritten, using the variable $q=2 r^{1 / 2}$ instead of $r$ in the first term, as

$$
H=2 \kappa_{2} \sqrt{\overline{u^{\prime 2}}(t)}\left(\frac{\partial^{2}}{\partial q^{2}}+\frac{9}{q} \frac{\partial}{\partial q}\right)+2 v\left(\frac{\partial^{2}}{\partial r^{2}}+\frac{4}{r} \frac{\partial}{\partial r}\right) .
$$

The operator $H$ admits a representation in the form of a sum of the so-called radial components of Laplace-Beltrami type operators [5] given on the Cartesian product $Z_{1} \times Z_{2}$, where $Z_{1}=\mathbb{R}_{q} \times S^{10}$ and $Z_{2}=\mathbb{R}_{r} \times S^{5}$ equipped with the corresponding 
metrics $d z_{1}^{2}$ and $d z_{2}^{2}$. The representation obtained for the operator $H$ gives reason to explore the property that $H$ generates a semigroup on some function space. This assertion was established by Grebenev and Oberlack [5] using the Trotter-Kato formula [17, p. 125] for pairs of continuous contractive semigroups. The basis of this approach is the theory of linear semigroups for the heat equation. Therefore, solutions of the problem (A.5)-(A.8) are defined by the formulas [5]

$$
B_{1 L L}(r, t) \equiv \tilde{v}(r, \tilde{\theta})=e^{-\tilde{\theta}(t) D_{\alpha}} B_{10 L L}(r), \quad B_{2 L L}(r, t) \equiv \hat{v}(r, \hat{\theta})=e^{-\hat{\theta}(t) B_{k}} B_{20 L L}(r),
$$

where $\tilde{\theta}$ and $\hat{\theta}$ are determined by

$$
d \tilde{\theta}=2 \kappa_{2} \sqrt{B_{1 L L}(0, t)} d t, \quad d \hat{\theta}=2 v d t, \quad \tilde{\theta}(0)=0, \quad \hat{\theta}(0)=0 .
$$

Here the operators $-D_{\alpha}$ and $-B_{k}$ generate continuous contractive semigroups $e^{-\tilde{\theta}(t) D_{\alpha}}$ and $e^{-\hat{\theta}(t) B_{k}}$ in the function space

$$
H_{0}^{\lambda}=\left\{F \in C_{0} \mid F \in h^{\lambda}([0, \infty))\right\}, \quad 0<\lambda<1,
$$

where $C_{0}$ is a set of bounded uniformly continuous functions given on $[0, \infty)$ such that $F(r) \rightarrow 0$ as $r \rightarrow \infty$ and $h^{\lambda}$ denotes the so-called little Hölder space. Then the formula

$$
S(t ; v)=\lim _{n \rightarrow \infty}\left(e^{-\tilde{\theta}(t / n) D_{\alpha}} \cdot e^{-\hat{\theta}(t / n) B_{k}}\right)^{n},
$$

known as the Trotter-Kato formula, defines the continuous contractive semigroup $S(t ; v)$ on the space $H_{0}^{s}, 0<s<1$, and

$$
\begin{aligned}
\frac{d}{d t} S(t ; v) B_{L L}(r, 0)=2 & \kappa_{2} \sqrt{\left.\left[S(t ; v) B_{L L}(r, 0)\right]\right|_{r=0}} D_{\alpha} S(t ; v) B_{L L}(r, 0) \\
+ & 2 v B_{k} S(t ; v) B_{L L}(r, 0) .
\end{aligned}
$$

Here the power $n$ means iteration; for example,

$$
\left(e^{-\tilde{\theta}(t / 2) D_{\alpha}} \cdot e^{-\hat{\theta}(t / 2) B_{k}}\right)^{2}=e^{-\tilde{\theta}(t / 2) D_{\alpha}} \cdot e^{-\hat{\theta}(t / 2) B_{k}} \cdot e^{-\tilde{\theta}(t / 2) D_{\alpha}} \cdot e^{-\hat{\theta}(t / 2) B_{k}} .
$$

The function obtained, $B_{L L}(r, t)=S(t ; v) B_{L L}(r, 0)$, is associated with the semigroup solution of the problem

$$
\begin{aligned}
\frac{\partial}{\partial t} B_{L L} & =\frac{1}{r^{4}} \frac{\partial}{\partial r} r^{4}\left(2 \kappa_{2} r \sqrt{B_{L L}(0, t)}+2 v\right) \frac{\partial}{\partial r} B_{L L}, \\
B_{L L}(0, r) & =B_{0 L L}(r) .
\end{aligned}
$$

Notice that in (A.9) the operators $e^{-\hat{\theta}(t / n) B_{k}}$ and $e^{-\tilde{\theta}(t / n) D_{\alpha}}$ admit permutation due to the inequality

$$
\left\|e^{-\hat{\theta}(t / n) B_{k}} e^{-\tilde{\theta}(t / n) D_{\alpha}}(\tilde{v})-e^{-\tilde{\theta}(t / n) D_{\alpha}} e^{-\hat{\theta}(t / n) B_{k}}(\tilde{v})\right\| \leq \frac{T \mu(t) t}{n},
$$

where $T$ is a positive constant, $\mu(t) \rightarrow 0$ as $t \rightarrow 0$, and $\|\cdot\|$ denotes the norm of $H_{0}^{s}$. The proof of (A.10) is via a result of Ebin and Marsden [3, Lemma 2, Appendix B]. 
It should be noted that the convergence in the formula (A.9) is of the order $O(1 / n)$ and independent of the viscosity $v$. Therefore,

$$
B_{L L}^{n}(r, t)=\left(e^{-\tilde{\theta}(t / n) D_{\alpha}} \cdot e^{-\hat{\theta}(t / n) B_{k}}\right)^{n} B_{L L}(r, 0)
$$

represents a smooth approximate solution and the error for large $n$ is $O(1 / n)$. Moreover, the iterations $\left(e^{-\tilde{\theta}(t / n) D_{\alpha}} \cdot e^{-\hat{\theta}(t / n) B_{k}}\right)^{n}$ converge in $H_{0}^{s}$ uniformly in $t$.

Now we prove that the semigroup $S(t ; v)$ admits the Loitsyansky integral invariant

$$
\int_{0}^{\infty} r^{4} S(t ; v) B_{L L}(r, 0) d r=\int_{0}^{\infty} r^{4} B_{L L}(r, 0) d r \equiv \Lambda<\infty .
$$

The proof is based on the explicit representation of the semigroups $e^{-\hat{\theta}(t / n) B_{k}}, e^{-\tilde{\theta}(t / n) D_{\alpha}}$. Let us consider the semigroup $e^{-\hat{\theta}(t / n) B_{k}}$. Then

$$
\hat{v}(r, \hat{\theta})=e^{-\hat{\theta} B_{k}} \hat{v}(r, 0) \equiv \int_{0}^{\infty} \xi^{4} G^{(1)}(r, \xi, \hat{\theta}) \hat{v}(\xi, 0) d \xi
$$

where

$$
G^{(1)}(r, \xi, \hat{\theta})=\frac{|r \xi|^{-3 / 2}}{4 \hat{\theta}} \exp \left(-\frac{r^{2}+\xi^{2}}{4 \hat{\theta}}\right) I_{3 / 2}\left(\frac{|r \xi|}{2 \hat{\theta}}\right) .
$$

Here $I_{m}$ denotes the modified Bessel function. Suppose that $r^{4} \hat{v}(r, \cdot) \in L_{1}\left(\mathbb{R}^{+}\right)$. Multiplying (A.11) by $r^{4}$ and then integrating over $r$,

$$
\int_{0}^{\infty} r^{4} \hat{v}(r, \hat{\theta}) d r=\int_{0}^{\infty} r^{4}\left(\int_{0}^{\infty} \xi^{4} G^{(1)}(r, \xi, \hat{\theta}) \hat{v}(\xi, 0) d \xi\right) d r
$$

We can rewrite this integral in the form

$$
\int_{0}^{\infty} \int_{0}^{\infty} r^{4} \xi^{4} G^{(1)}(r, \xi, \hat{\theta}) \hat{v}(\xi, 0) d \xi d r=\int_{0}^{\infty} \xi^{4} v(\xi, 0)\left(\int_{0}^{\infty} r^{4} G^{(1)}(r, \xi, \hat{\theta}) d r\right) d \xi .
$$

Using the symmetry of $G^{(1)}(r, \xi, \hat{\theta})$ with respect to $r, \xi$ and the equality

$$
\int_{0}^{\infty} \xi^{4} G^{(1)}(r, \xi, \hat{\theta}) d \xi=1,
$$

we deduce that the interior integral in (A.12) equals 1 , which yields

$$
\int_{0}^{\infty} r^{4} \hat{v}(r, \hat{\theta}) d r=\int_{0}^{\infty} \xi^{4} \hat{v}(\xi, 0) d \xi \equiv \int_{0}^{\infty} r^{4} \hat{v}(r, 0) d r .
$$

Hence the semigroup $e^{-\hat{\theta} B_{k}}$ admits the Loitsyansky integral invariant. To prove this property for the semigroup $e^{-\tilde{\theta} D_{\alpha}}$, we use the fact that the operator $D_{\alpha}$ is transformed into the operator $B_{k}$ by the change of variable $q=2 \sqrt{r}$. Thus

$$
\tilde{v}(r, \tilde{\theta}) \equiv \tilde{u}(q, \tilde{\theta})=e^{-\tilde{\theta} B_{k}} \tilde{u}(q, 0) \equiv \int_{0}^{\infty} \tilde{\xi}^{9} G^{(2)}(q, \tilde{\xi}, \tilde{\theta}) \tilde{u}(\tilde{\xi}, 0) d \tilde{\xi} \quad \text { for } k=9
$$


where

$$
G^{(2)}(q, \tilde{\xi}, \tilde{\theta})=\frac{|q \tilde{\xi}|^{-4}}{4 \tilde{\theta}} \exp \left(-\frac{q^{2}+\tilde{\xi}^{2}}{4 \tilde{\theta}}\right) I_{4}\left(\frac{|q \tilde{\xi}|}{2 \tilde{\theta}}\right) .
$$

Using the same argument,

$$
\int_{0}^{\infty} q^{9} \tilde{u}(q, \tilde{\theta}) d q=\int_{0}^{\infty} \tilde{\xi}^{9} \tilde{u}(\tilde{\xi}, 0) d \tilde{\xi}
$$

or, in the original variable $r$,

$$
\int_{0}^{\infty} r^{4} \tilde{v}(r, \tilde{\theta}) d r=\int_{0}^{\infty} \xi^{4} \tilde{v}(\xi, 0) d \xi \equiv \int_{0}^{\infty} r^{4} \tilde{v}(r, 0) d r .
$$

The invariants (A.13) and (A.14) of the semigroups $e^{-\tilde{\theta} D_{\alpha}}$ and $e^{-\hat{\theta} B_{k}}$, which have the same functional form, are crucial for proving that $S(t ; v)$ admits the Loitsyansky integral invariant.

To start with, we prove this property for the $n$ th-order approximation

$$
S^{n}(t, v)=\left(e^{-\tilde{\theta}(t / n) D_{\alpha}} \cdot e^{-\hat{\theta}(t / n) B_{k}}\right)^{n}
$$

of the semigroup $S(t, v)$. Here

$$
\left(e^{-\tilde{\theta}(t / n) D_{\alpha}} \cdot e^{-\hat{\theta}(t / n) B_{k}}\right)^{n}=\overbrace{2 \text { terms }}^{\overbrace{\underbrace{-\tilde{\theta}(t / n) D_{\alpha}} \cdot e^{-\hat{\theta}(t / n) B_{k}}}^{n \text { pairs }} \cdots \cdot e^{-\tilde{\theta}(t / n) D_{\alpha}} \cdot e^{-\hat{\theta}(t / n) B_{k}}} .
$$

From this representation it follows that it is sufficient to show that the composition of the two semigroups $e^{-\tilde{\theta}(t / n) D_{\alpha}}$ and $e^{-\hat{\theta}(t / n) B_{k}}$ admits the Loitsyansky integral invariant. Let us consider

$$
B_{L L}^{n}(r, \cdot)=e^{-\tilde{\theta}(t / n) D_{\alpha}} \cdot e^{-\hat{\theta}(t / n) B_{k}} B_{L L}^{n-1}(r, \cdot) .
$$

Multiplying by $r^{4}$ and integrating over $r$ for all $t>0$,

$$
\begin{aligned}
\int_{0}^{\infty} r^{4} B_{L L}^{n}(r, \cdot) d r & =\frac{1}{2^{9}} \int_{0}^{\infty} q^{9} \tilde{u}(q, \cdot) d q \\
& =\frac{1}{2^{9}} \int_{0}^{\infty} q^{9}\left(\int_{0}^{\infty} \tilde{\xi}^{9} G^{(2)}(q, \tilde{\xi}, \cdot) \tilde{u}(\tilde{\xi}, \cdot) d \tilde{\xi}\right) d q \\
& =\frac{1}{2^{9}} \int_{0}^{\infty} \tilde{\xi}^{9} \tilde{u}(\tilde{\xi}, \cdot)\left(\int_{0}^{\infty} q^{9} G^{(2)}(q, \tilde{\xi}, \cdot) d q\right) d \tilde{\xi}
\end{aligned}
$$

where $\tilde{u}(q, \cdot)=B_{L L}^{n}(r, \cdot)$ for $q=2 r^{1 / 2}$. Since

$$
\int_{0}^{\infty} \xi^{9} G^{(2)}(q, \tilde{\xi}, \cdot) d \tilde{\xi}=1
$$

and $G^{(2)}(q, \tilde{\xi}, \cdot)$ is a symmetric function with respect to $q$ and $\tilde{\xi}$, we deduce again that the interior integral equals 1 . Hence

$$
\int_{0}^{\infty} r^{4} B_{L L}^{n}(r, \cdot) d r=\frac{1}{2^{9}} \int_{0}^{\infty} \tilde{\xi}^{9} \tilde{u}(\tilde{\xi}, \cdot) d \tilde{\xi}=\int_{0}^{\infty} \xi^{4} \tilde{v}(\xi, \cdot) d \xi
$$


where $\tilde{v}(\xi, \cdot)=\tilde{u}(\tilde{\xi}, \cdot)$ for $\tilde{\xi}=2 \xi^{1 / 2}$. This in turn implies that

$$
\int_{0}^{\infty} \xi^{4} \tilde{v}(\xi, \cdot) d \xi=\int_{0}^{\infty} r^{4} \tilde{v}(r, \cdot) d r=\int_{0}^{\infty} r^{4}\left(\int_{0}^{\infty} \xi^{4} G^{(1)}(r, \xi, \cdot) B_{L L}^{n-1}(\xi, \cdot) d \xi\right) d r .
$$

Therefore,

$$
\int_{0}^{\infty} r^{4} B_{L L}^{n}(r, \cdot) d r=\int_{0}^{\infty} r^{4} B_{L L}^{n-1}(r, \cdot) d r
$$

To complete the proof we iterate this procedure $n$ times. This yields

$$
\int_{0}^{\infty} r^{4} B_{L L}^{n}(r, t) d r=\int_{0}^{\infty} r^{4} B_{L L}(r, 0) d r .
$$

From (A.15) we infer that $r^{4} B_{L L}^{n}(r, \cdot) \in L_{1}\left(\mathbb{R}^{+}\right)$if $r^{4} B_{L L}(r, 0)$ is an integrable function and $S^{n}(t, v)$ preserves the Loitsyansky invariant. We observe that $\left\{B_{L L}^{n}(r, t)\right\}$ is a sequence of nonnegative smooth functions which converges to a nonnegative continuous function

$$
B_{L L}(r, t)=S(t, v) B_{L L}(r, 0)=\lim _{n \rightarrow \infty}\left(e^{-\tilde{\theta}(t / n) D_{\alpha}} \cdot e^{-\hat{\theta}(t / n) B_{k}}\right)^{n} B_{L L}(r, 0)
$$

in the norm of $H_{0}^{s}$. Positivity of $B_{L L}(r, t)$ follows from the inequalities $e^{-\tilde{\theta}(t / n) D_{\alpha}}>0$ and $e^{-\hat{\theta}(t / n) B_{k}}>0$. Convergence in $H_{0}^{s}$ implies the uniform convergence of the sequence $\left\{B_{L L}^{n}(r, \cdot)\right\}$ on each compact subset of $\mathbb{R}^{+}$and therefore the pointwise convergence of $\left\{B_{L L}^{n}(r, \cdot)\right\}$ on $\mathbb{R}^{+}$. Hence $\left\{r^{4} B_{L L}^{n}(r, \cdot)\right\}$ is a pointwise convergent sequence of integrable nonnegative functions. Using Fatou's lemma from functional analysis, we obtain

$$
\int_{0}^{\infty} r^{4} B_{L L}(r, \cdot) d r \leq \lim _{n \rightarrow \infty} \inf \int_{0}^{\infty} r^{4} B_{L L}^{n}(r, \cdot) d r \equiv \Lambda,
$$

or $r^{4} B_{L L}(r, \cdot) \in L_{1}\left(\mathbb{R}^{+}\right) \cap C_{0}\left(\mathbb{R}^{+}\right)$. Now notice that

$$
r^{4} B_{L L}^{n}(r, \cdot) \leq A / x^{1+\beta} \quad \text { and } \quad r^{4} B_{L L}(r, \cdot) \leq A / x^{1+\beta}
$$

for $r>K, \beta>0$ (for some positive numbers $A, K$ ), in view of the fact that both $r^{4} B_{L L}^{n}(r, \cdot)$ and $r^{4} B_{L L}(r, \cdot)$ are integrable functions. Let us estimate

$$
\begin{aligned}
\left|\int_{0}^{\infty} r^{4} B_{L L}(r, \cdot) d r-\int_{0}^{\infty} r^{4} B_{L L}^{n}(r, \cdot) d r\right| \\
\leq\left|\int_{0}^{b_{m}} r^{4} B_{L L}(r, \cdot) d r-\int_{0}^{b_{m}} r^{4} B_{L L}^{n}(r, \cdot) d r\right| \\
+\left|\int_{b_{m}}^{\infty} r^{4} B_{L L}(r, \cdot) d r\right|+\left|\int_{b_{m}}^{\infty} r^{4} B_{L L}^{n}(r, \cdot) d r\right| .
\end{aligned}
$$

Choosing $b_{m}>K$ gives

$$
\left|\int_{b_{m}}^{\infty} r^{4} B_{L L}(r, \cdot) d r\right|+\left|\int_{b_{m}}^{\infty} r^{4} B_{L L}^{n}(r, \cdot) d r\right|<\frac{\delta}{2}
$$


for arbitrary small $\delta>0$. Note that $r^{4} B_{L L}^{n}(r, \cdot) \rightarrow r^{4} B_{L L}(r, \cdot)$ as $n \rightarrow \infty$ uniformly on each compact subset of $\mathbb{R}^{+}$. Hence

$$
\left|\int_{0}^{b_{m}} r^{4} B_{L L}(r, \cdot) d r-\int_{0}^{b_{m}} r^{4} B_{L L}^{n}(r, \cdot) d r\right| \leq \frac{\delta}{2}
$$

for sufficiently large $n$. Therefore,

$$
\left|\int_{0}^{\infty} r^{4} B_{L L}(r, \cdot) d r-\int_{0}^{\infty} r^{4} B_{L L}^{n}(r, \cdot) d r\right| \leq \delta
$$

and

$$
\lim _{n \rightarrow \infty} \int_{0}^{\infty} r^{4} B_{L L}^{n}(r, \cdot) d r=\int_{0}^{\infty} r^{4} B_{L L}(r, \cdot) d r .
$$

Thus, we have shown that the semigroup $S(t, v)$ admits the Lointsyansky integral invariant.

\section{References}

[1] G. Birkhoff, "Fourier synthesis of homogeneous turbulence", Comm. Pure Appl. Math. 7 (1954) 19-44, doi:10.1002/cpa.3160070104.

[2] A. F. Cheviakov, "GeM: a Maple module for symmetry and conservation law computation for PDEs/ODEs", http://math.usask.ca/ cheviakov/gem/.

[3] D. G. Ebin and J. Marsden, "Groups of diffeomorphisms and the motion of an incompressible fluid”, Ann. of Math. Ser. 292 (1970) 102-163, doi:10.2307/1970699.

[4] A. Erdélyi (ed.) Higher transcendental functions, Volume I (Bateman Manuscript Project) (McGraw-Hill, New York, 1953).

[5] V. N. Grebenev and M. Oberlack, "A Chorin-type formula for solutions to a closure model for the von Kármán-Howarth equation”, J. Nonlinear Math. Phys. 12 (2005) 1-9, doi:10.2991/jnmp.2005.12.1.1.

[6] V. N. Grebenev and M. Oberlack, "A geometric interpretation of the second-order structure function arising in turbulence", Math. Phys. Anal. Geom. 12 (2008) 1-18, doi:10.1007/s11040-008-9049-4.

[7] D. Hurst and J. C. Vassilicos, "Scalings and decay of fractal-generated turbulence", Phys. Fluids 19 (2007) 035103, doi:10.1063/1.2676448.

[8] N. H. Ibragimov, A practical course in differential equations and mathematical modelling (ALGA Publications, Karlskrona, Sweden, 2005).

[9] T. von Kármán and L. Howarth, "On the statistical theory of isotropic turbulence", Proc. R. Soc. Lond. A 164 (1938) 192-215, doi:10.1098/rspa.1938.0013.

[10] S. J. Liao, "An explicit analytic solution to the Thomas-Fermi equation", Appl. Math. Comput. 144 (2003) 495-506, doi:10.1016/S0096-3003(02)00423-X.

[11] S. J. Liao, Beyond perturbation: introduction to the homotopy analysis method (Chapman \& Hall/CRC, Boca Raton, FL, 2004).

[12] S. J. Liao, "Notes on the homotopy analysis method: some definitions and theorems", Commun. Nonlinear Sci. Numer. Simul. 14 (2009) 983-997, doi:10.1016/j.cnsns.2008.04.013.

[13] S. J. Liao, "On the relationship between the homotopy analysis method and Euler transform", Commun. Nonlinear Sci. Numer. Simul. 15 (2010) 1421-1431, doi:10.1016/j.cnsns.2009.06.008.

[14] S. J. Liao, "An optimal homotopy-analysis approach for strongly nonlinear differential equations", Commun. Nonlinear Sci. Numer. Simul. 15 (2010) 2003-2016, doi:10.1016/j.cnsns.2009.09.002.

[15] S. J. Liao and K. F. Cheung, "Homotopy analysis of nonlinear progressive waves in deep water", J. Engrg. Math. 45 (2003) 105-116, doi:10.1023/A:1022189509293. 
[16] L. G. Loitsyansky, "Some basic laws of isotropic turbulent flow", Report no. 440, Central AeroHydrodynamical Institute, Moscow, 1939.

[17] J. Marsden, Applications of global analysis in mathematical physics (Publish or Perish, Inc., Berkeley, CA, 1974).

[18] A. S. Michenko and A. T. Fomenko, Lectures on differential geometry and topology (Factorial Press, Moscow, 2000).

[19] A. S. Monin and A. M. Yaglom, Statistical hydromechanics (Gidrometeoizdat, St. Petersburg, 1994).

[20] M. Oberlack, "On the decay exponent of isotropic turbulence", Proc. Appl. Math. Mech. (PAMM) 1 (2002) 294-297, doi:10.1002/1617-7061(200203)1:1<294::AID-PAMM294>3.0.CO;2-W.

[21] M. Oberlack and N. Peters, "Closure of the two-point correlation equation as a basis for Reynolds stress models", Appl. Sci. Res. 51 (1993) 533-538, doi:10.1007/BF01082587.

[22] M. Oberlack and N. Peters, "Closure of the two-point correlation equation in physical space as a basis for Reynolds stress models", in: Near-wall turbulent flows (eds R. M. C. So, C. G. Speziale and B. E. Launder), (Elsevier Science, Amsterdam, 1993) 85-94.

[23] M. Oberlack and A. Rosteck, "New statistical symmetries of the multi-point equations and its importance for turbulent scaling laws", Discrete Contin. Dyn. Syst. Ser. S 3 (2010) 451-471, doi:10.3934/dcdss.2010.3.451.

[24] J. Piquet, Turbulent flows: models and physics (Springer, Berlin, 2010).

[25] L. Roman-Miller and P. Broadbridge, "Exact integration of reduced Fisher's equation, reduced Blasius equation, and the Lorenz model", J. Math. Anal. Appl. 251 (2000) 65-83, doi:10.1006/jmaa.2000.7020.

[26] A. Rosteck and M. Oberlack, "Lie algebra of the symmetries of the multi-point equations in statistical turbulence theory", J. Nonlinear Math. Phys. 18 (2011) 251-264, (Supplementary Issue 1), doi:10.1142/S1402925111001404.

[27] R. E. Seoud and J. C. Vassilicos, "Dissipation and decay of fractal-generated turbulence", Phys. Fluids 19 (2007) 105108, doi:10.1063/1.2795211.

[28] R. W. Stewart and A. A. Townsend, "Similarity and self-preservation in isotropic turbulence", Philos. Trans. R. Soc. Lond. A 243 (1951) 359-386, doi:10.1098/rsta.1951.0007. 